closing the vaginal cuff as the first step of the minimally invasive surgery. However, for patients with the Ia1 stage, there is no need for extensive vaginal margin. Moreover, performing laparoscopic hysterectomy without a uterine manipulator is challenging. In this context, vNOTES provides an easy solution.

Description We performed a cervical cerclage invaginating the external cervix orifice followed by conventional vNOTES hysterectomy with bilateral salpingectomy. The patient was positioned in stirrups in Trendelenburg position and standard sterilization was performed. After cervical cerclage, a circular incision was made around the uterine cervix and the following structures were sealed and divided by an advanced bipolar device: uterosacral ligaments, anterior bladder pillars, parametria. Then, a self-constructed vaginal port with alexis ${ }^{\circledR}$ and surgical glove was inserted through the vagina. Pneumoperitoneum was inflated and the sealing/division of uterine arteries, round ligaments, ovarian ligaments, and broad ligaments were completed.

Conclusion vNOTES may provide a safe minimally invasive hysterectomy for Ia1 cervical carcinoma.

\section{IGCS20 1115}

\section{LATERALLY EXTENDED PARAMETRECTOMY (LEP)}

M Capilna*, SL Kiss, A Cozlea, M Stanca, M Gheorghe. First Obstetrics and Gynecology Clinic, 'G. E. Palade' University of Medicine, Pharmacy, Science and Technology, Romania

10.1136/ijgc-2020-IGCS.407

Introduction Laterally Extended Parametrectomy (LEP) was imagined by Ungar and Palfalvi as a more radical surgical procedure for the treatment of lymph node positive stage $\mathrm{Ib}$ and stage IIb cervical cancer.

Methods The aim of the technique is to remove the entire parametrial tissue containing lymphatic structures from the pelvic side wall.

Results LEP superposes to a type D Querleu-Morrow radical hysterectomy, extending the lateral limits of the dissection not only to the medial surface of hypogastric vessels, but to true borders of the pelvic side wall. Its rationale was to avoid the need of aggresive and deleterious postoperative radiotherapy for patients with positive pelvic lymph nodes or parametria in which the final histology suggested a complete removal of the potentially tumor containing lymphvessel and lymph node containing fibro-fatty tissue. LEP may be also taken into consideration during pelvic exenteration, when the tumor involves the soft structures of the pelvic side wall, for a more extensive pelvic side wall dissection.

During LEP, together with the visceral branches of hypogastric vessels, all the parietal branches are also divided (ilio-lumbal, obturator, gluteal superior and inferior and internal pudendal vessels) at the level where the vessels leave or enter into the pelvis. LEP can be performed on one or both pelvic sides, depending on parametrial invasion or presence positive lymph nodes uni- or bilaterally.

Conclusion LEP provides a good chance for survival without the toxicity of radiotherapy for pelvic lymph node positive stage Ib or IIb cervical cancer patients.
IGCS20_1321

\section{LAPAROSCOPIC PRIMARY REPAIR OF DUODENAL PERFORATION AFTER LAPAROSCOPIC PARA-AORTIC LYMPHADENECTOMY}

JS Choi* . Hanyang University College of Medicine, South Korea

\subsection{6/ijgc-2020-IGCS.408}

Objective To present of laparoscopic primary repair of duodenal perforation after laparoscopic para-aortic lymphadenectomy for the patient with endometrial carcinoma.

Patients A 78-year-old woman with postmenopausal bleeding and thickened endometrium presented to our department. The histopathology of biopsied endometrium revealed grade 1 endometrioid adenocarcinoma. The MRI shows an about 5 $\mathrm{cm}$ sized tumor within the endometrial cavity suspicious myometrial invasion.

Interventions We perform the laparoscopic staging surgery. No intraoperative complications were recognized. However, on postoperative day 1 , the color of intra-abdominal drainage change from serosanginous to dark green. We strongly suspected small bowel perforation and perform secondary laparoscopic surgery immediately. We scrutinized the small bowel and found the perforation site on duodenum. The perforation occurred at the horizontal part of duodenum ventrally vena cava. We carried out laparoscopic primary repair with 3-0 vicryl. Double layer closure was done by interrupted suture in first layer and Lambert suture for second layer. Then, we placed drainage into the duodenal repair site and traced the small bowel meticulously. We reviewed the video of primary surgery. We thought that the thermal injury was occurred by ultrasonic cutting and coagulating device during the lymphadenectomy in pre-caval area just below duodenum or mechanical micro-perforation is made during lifting the duodenum by dissecting forcep. After duodenal repair, endoscopically guided placement of nasogastric tube was performed. Gastrography did not show any leakage at the site of duodenal repair on postoperative day 3 .

Conclusions Immediate laparoscopic primary repair of duodenal perforation after laparoscopic para-aortic lymphadenectomy is safe and feasible.

\section{IGCS20 1469}

\section{LAPAROSCOPIC RESECTION OF BULKY PARA-AORTIC LYMPH NODE METASTASIS}

JS Choi* . Hanyang University College of Medicine, South Korea

\subsection{6/ijgc-2020-IGCS.409}

Objective To present of laparoscopic resection of bulky paraaortic lymph node metastasis discovered during laparoscopic restaging surgery for unexpected ovarian malignancy

Patients A 45-year-old woman with prior laparoscopic bilateral salpingo-oophorecetomy, presented to our department with unexpected ovarian malignancy which was resulted from the high grade serous carcinoma. Preoperative PET CT scan shows enlarged lymph node in aorto-caval area and no abnormal finding in peritoneal cavity and previous operative site. 